\title{
LE SYSTÈME DE SURVEILLANCE DE L'ÉTAT SANITAIRE DE LA FORÊT EN FRANCE
}

\author{
Ch. BARTHOD
}

De septembre 1987 à décembre 1990, au terme d'une réflexion entreprise en 1984, le ministère de l'Agriculture et de la Forêt a pris une série de décisions visant à doter la France d'un système cohérent de surveillance de l'état sanitaire des forêts, sur un modèle à trois niveaux qui s'est progressivement imposé à la plupart des grands pays forestiers européens. Le dispositif, désormais complet, repose sur trois réseaux répondant à des objectifs complémentaires.

\section{LE RÉSEAU DE CORRESPONDANTS-OBSERVATEURS}

La réorganisation profonde du dispositif antérieur de surveillance phytosanitaire, mis en place et animé par le CEMAGREF, depuis le début des années 1970, avait été voulue par Messieurs Francis Rinville, directeur des Forêts, et Jean-François Lacaze (INRA-CEMAGREF) dès 1984. Au terme de plus de trois ans de concertation entre l'ensemble des partenaires intéressés et d'un bilan des dispositifs gérés par quelques grands pays forestiers européens et nord-américains, le nouveau dispositif français a été adopté par le ministère de l'Agriculture et de la Forêt le 24 septembre 1987. Son animation technique est assumée par le Département de la Santé des Forêts (DSF, service technique du ministère de l'Agriculture, directement rattaché au directeur de l'Espace rural et de la Forêt), qui mobilise à cet effet un budget annuel de fonctionnement d'environ 3 millions de francs (hors salaires) pour l'ensemble du réseau (y compris les indemnisations des organismes parties prenantes). L'ensemble du dispositif et les missions qui lui sont confiées sont décrits dans la circulaire DERF/N88/n 3008 du 10 mai 1988.

Le premier objectif de ce réseau est de repérer le plus précocement possible les fluctuations de population des principaux insectes ravageurs ou d'épidémies des principaux champignons pathogènes que la bibliographie et l'expérience des entomologistes et pathologistes de I'INRA et du CEMAGREF ont identifiés comme présentant un danger potentiel. Ce repérage précoce doit permettre l'information des gestionnaires avant que le problème ne soit au maximum de son ampleur, ainsi que l'élaboration d'une stratégie de gestion du risque phytosanitaire identifié. Celleci doit prendre en compte les objectifs et les contraintes du propriétaire, à un moment où une éventuelle intervention peut encore permettre d'éviter des dégâts dépassant le seuil acceptable localement. $\dot{A}$ terme, pour certains problèmes majeurs, il serait souhaitable d'arriver à une prognose. II est nécessaire pour cela de constituer une véritable mémoire phytosanitaire, suffisamment précise quant aux différents paramètres qui influent sur la manifestation du problème. Le second objectif est de situer correctement la fréquence et l'impact des accidents phytosanitaires sur la gestion, grâce à des données spatiales et temporelles. Cette "relativisation " des problèmes phytosanitaires doit permettre aux gestionnaires de mieux les intégrer dans leurs analyses et leurs choix, en terme de risque normal. Là aussi, la constitution progressive d'une mémoire phytosanitaire est indispensable. 
Organisé en un échelon central et cinq échelons techniques interrégionaux (Nancy, Orléans, Bordeaux, Clermont-Ferrand et Avignon), le Département de la Santé des Forêts emploie 9 ingènieurs, 7 techniciens et 6 secrétaires. II anime un important réseau d'environ 230 correspondantsobservateurs, qui constitue la partie la plus originale du dispositif. Volontaires recrutès au sein de l'ensemble des services et organismes forestiers [Office national des Forêts (ONF), Centre régional de la Propriété forestière (CRPF), Direction régionale de l'Agriculture et de la Forêt (DRAF), Direction départementale de l'Agriculture et de la Forêt (DDAF) ], recevant une formation continue régulière et travaillant environ trente jours par an dans le cadre défini par le Département de la Santé des Forêts, les correspondants-observateurs du DSF assurent, depuis janvier 1989, la surveillance sanitaire permanente de la forêt française métropolitaine, pour tous les problèmes entomologiques, pathologiques ou d'origine abiotique (feu excepté). La densitè moyenne du réseau est d'un correspondant-observateur pour 60000 hectares, mais elle est relativement inégale suivant les régions: plus forte là où la proportion de forêts soumises au régime forestier est plus élevée, plus faible dans les régions où la forêt privée est nettement prépondérante, malgré une

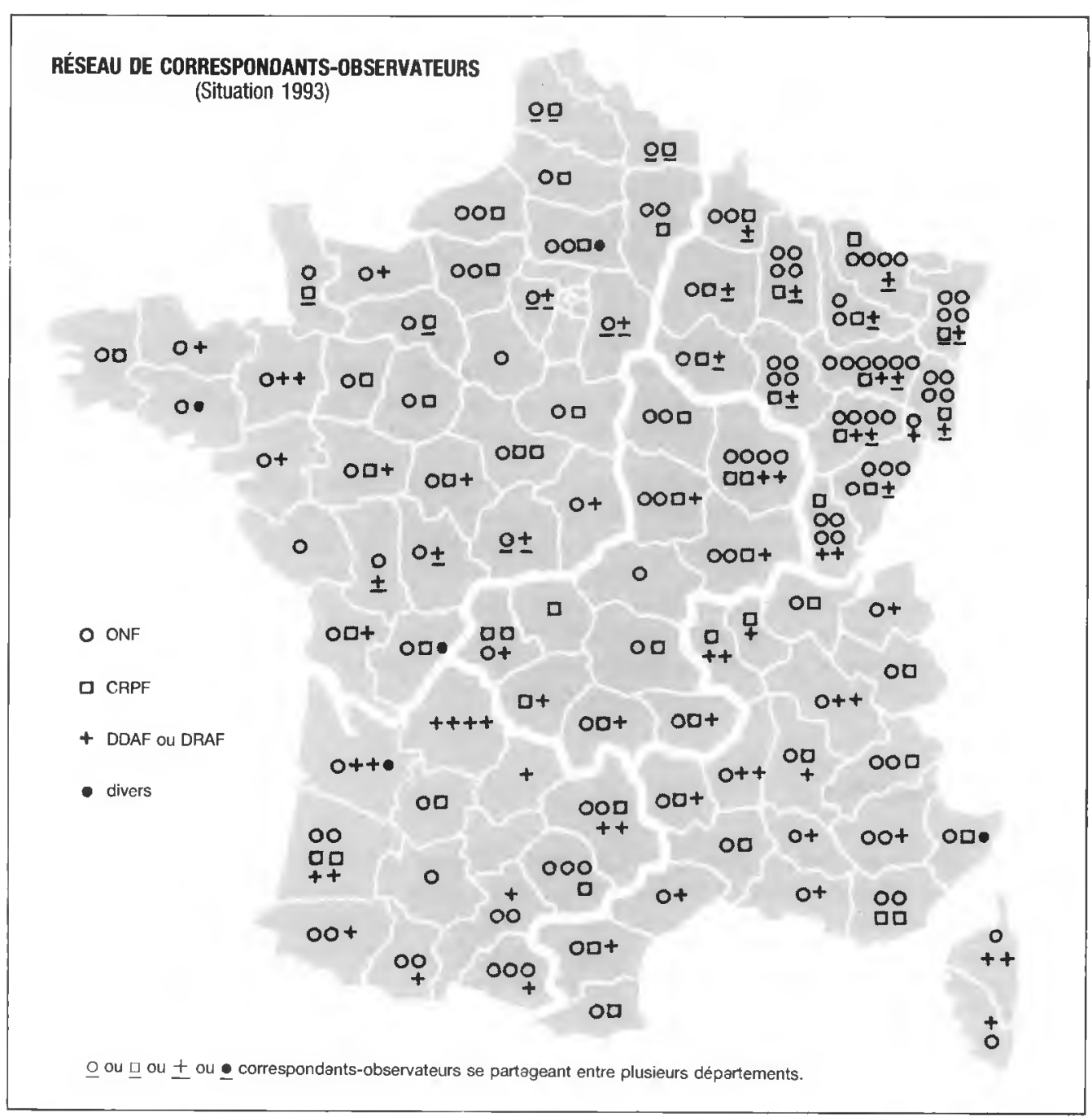




\section{Ch. BARTHOD}

remarquable mobilisation des CRPF. La transmission des fiches d'observation entre les correspondants-observateurs et les échelons techniques interrégionaux du DSF se fait essentiellement par voie télématique; après validation, ces données sont intégrées dans une base de données informatique.

Pour une cinquantaine d'insectes ravageurs et de champignons pathogènes que l'expérience conduit à considérer comme devant faire l'objet d'une attention prioritaire, des méthodologies spécifiques de surveillance et d'estimation quantitative des niveaux de population ont été élaborées. La plupart se mettent en œuvre à l'avancement ; d'autres utilisent des placettes permanentes (chenille processionnaire du Pin, tordeuse verte, géométrides, ...). Répertoriées dans le Manuel du correspondant-observateur du DSF, elles dérivent pour la plupart des méthodes équivalentes anglo-saxonnes et germaniques, mais aussi de l'expérience acquise par le CEMAGREF et par le Service québécois de protection contre les insectes et les maladies. Les problèmes nouveaux ou particulièrement complexes conduisent le Département de la Santé des Forêts à faire appel au Programme "Protection phytosanitaire de la forêt" du CEMAGREF, actuellement dirigé par Monsieur Claude-Bernard Malphettes et éventuellement, dans un deuxième temps, aux experts de I'INRA ou d'autres organismes spécialisés. Par ailleurs, environ $15 \%$ des problèmes observés concernent des dépérissements localisés d'origine complexe, où les insectes ravageurs et les champignons pathogènes ne semblent jouer qu'un rôle secondaire. Une première esquisse de méthodologie d'approche de ces dépérissements a été élaborée par Monsieur Louis-Michel Nageleisen, correspondant thématique du DSF pour les dépérissements.

Chaque année, environ 1400 échantillons sont déterminés [avec l'assistance du CEMAGREF pour les déterminations entomologiques les plus difficiles et du Laboratoire de Pathologie des Ligneux du Service régional de la Protection des Végétaux de Nancy (SRPV), dirigé par Monsieur Pierre Chandelier]. En plus des relevés sur placettes permanentes, près de 5500 observations de terrain sont faites. L'assistance technique aux correspondants-observateurs et aux gestionnaires forestiers représente plus de 1000 jours de terrain par an (et plus de $370000 \mathrm{~km}$ parcourus). Une quarantaine de bulletins d'avertissement ou d'information sont diffusés annuellement, sans compter la publication annuelle du bilan La santé des forêts en 19.. et la parution à périodicité irrégulière de la Lettre du DSF destinée essentiellement aux correspondants-observateurs.

Au terme de cinq années de montée en puissance, le réseau de correspondants-observateurs paraît donner satisfaction aux partenaires forestiers, ainsi que l'a mis en évidence le rapport d'évaluation du Département de la Santé des Forêts remis au ministre de l'Agriculture en 1993, conjointement par le Conseil général du GREF et le Conseil général d'Agronomie. Il se posera néanmoins à relativement brève échéance un problème pour la surveillance sanitaire de la forêt privée, avec le désengagement progressif des DDAF à la suite des réductions d'effectifs qui les frappent. Or, le réseau comporte environ un quart de correspondants-observateurs de DDAF et de DRAF, au côté d'un quart venant de CRPF ou autres organismes de la forêt privée et d'une moitié issue de l'ONF.

\section{LE RÉSEAU EUROPÉEN}

Au début des années 1980, un vif débat international avait porté sur les effets de la pollution atmosphérique à longue distance sur les forêts. Pour tenter de quantifier globalement l'état de vitalité des peuplements, estimé grossièrement par l'appréciation visuelle de la perte de feuilles et de la coloration anormale du houppier, le réseau implanté par l'ONF dans les Vosges en 1983 avait été progressivement étendu à une dizaine de grands massifs forestiers, couvrant au total 3 millions d'hectares boisés à partir de 1987, essentiellement dans l'Est de la France et en moyenne montagne. Ce réseau national, appelé réseau bleu, a fonctionné en France jusqu'à l'automne 1993. Les données ainsi accumulées durant onze années feront prochainement l'objet d'une exploitation aussi poussée que possible. 
Dans le cadre de la convention internationale sur la pollution atmosphérique transfrontière signée à Genève en 1979, un groupe de travail sous présidence allemande s'est efforcé d'harmoniser, à partir de 1985, les protocoles nationaux des réseaux d'observation d'une vingtaine de pays du continent européen. En 1986, sur la base de ces premières recommandations, les douze pays de la Communauté économique européenne ont négocié un protocole communautaire devant s'appliquer à l'ensemble des forêts de la CEE. Celui-ci repose sur un réseau systématique de placettes de 20 arbres implantées aux nœuds d'une grille à maille carrée de $16 \mathrm{~km}$ de côté. Les négociateurs français étaient Messieurs Pierre Bazire (IFN) et Pierre Martinot-Lagarde (ONF). L'accord trouvé au sein du Comité permanent forestier de la CEE a été rendu obligatoire par un règlement communautaire portant sur la protection des forêts communautaires contre les effets de la pollution atmosphérique.

Ce réseau systématique, appelé réseau européen, vise à donner une idée statistiquement représentative des grandes évolutions interannuelles qui affectent la vitalité des forêts européennes, sur la base d'un protocole harmonisé, sans préjuger des mécanismes éventuels en cause. Lors de la première conférence ministérielle pour la protection des forêts en Europe (Strasbourg, 1990), les

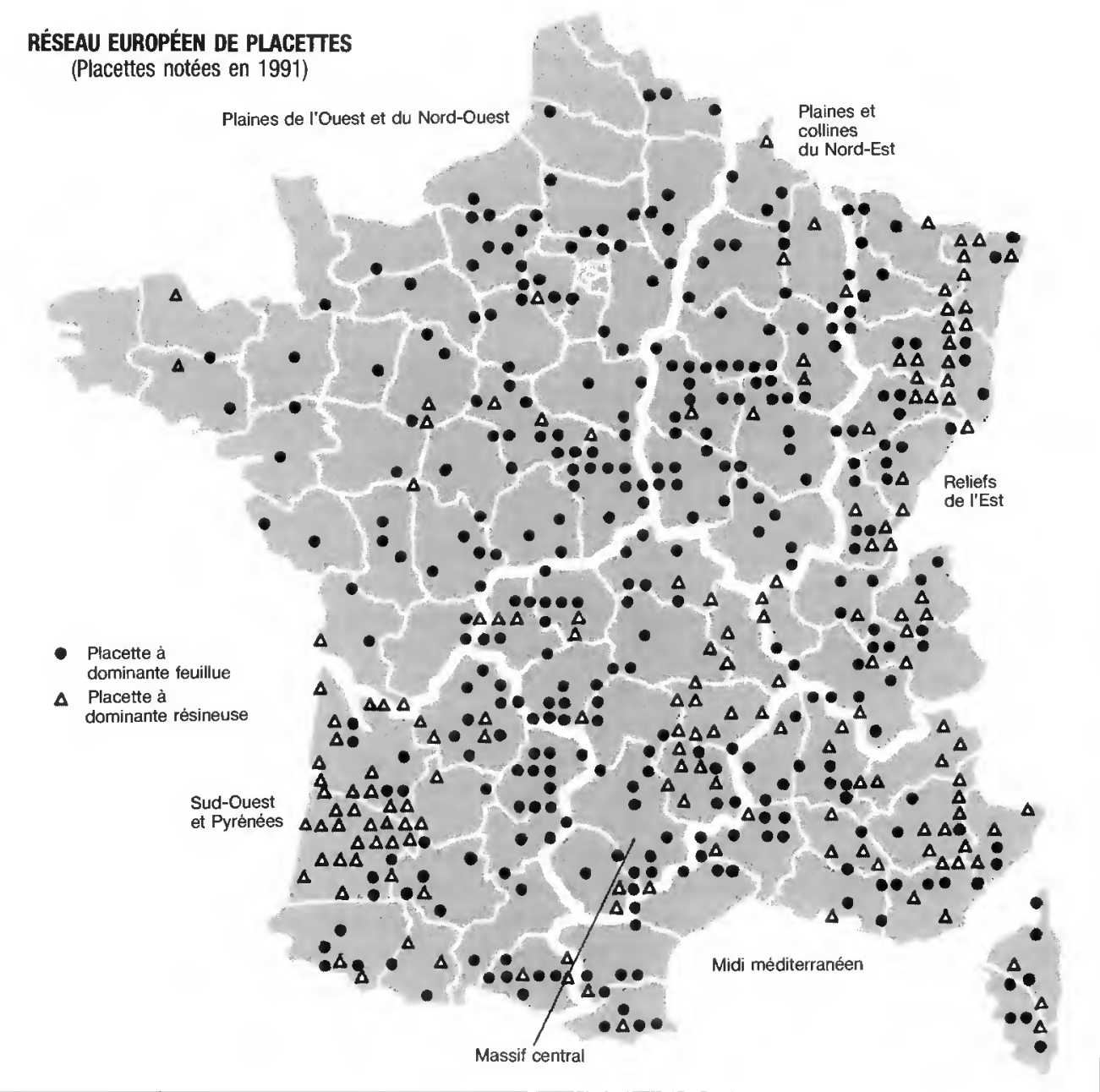




\section{Ch. BARTHOD}

trente-et-un pays du continent européen, dans leur première résolution, ont assigné à ce type de réseau l'objectif de permettre des bilans et suivis régionaux, sur une base statistique, de quelques paramètres simples concernant les conditions stationnelles et la vitalité des arbres, en cherchant à mieux appréhender la variabilité géographique et temporelle des paramètres mesurés et à assurer la comparabilité des données à l'échelle européenne.

La partie française de ce réseau a été mise en place par la note de service DERF/N88/ ${ }^{\circ} 3008$ du 3 mai 1988. Il est vraiment opérationnel depuis 1989 et compte environ 520 placettes. Géré par le Département de la Santé des Forêts, avec le concours de 6 formateurs de l'ONF et la participation de notateurs de l'ONF, des CRPF, des DRAF et des DDAF, ce réseau est appelé à s'articuler de plus en plus avec le réseau de correspondants-observateurs du DSF, grâce à l'incorporation d'un correspondant-observateur dans chaque équipe de deux notateurs du réseau européen. Le traitement informatique des données est assuré conjointement par le CEMAGREF et le DSF. L'interprétation des données est effectuée par deux ingénieurs du DSF, essentiellement à partir des évolutions interannuelles, car les notations en valeur absolue doivent être manipulées avec une grande prudence. Le coût annuel de fonctionnement de ce réseau est d'environ 1,2 million de francs (hors salaires).

Au sein des groupes de travail ad hoc du continent européen et de l'Union européenne, une attention grandissante est portée aux facteurs du milieu, notamment au sol. Le principal objectif poursuivi est d'arriver, à terme, à distinguer parmi les variations de l'état sanitaire observées sur plusieurs années, celles qui s'expliquent simplement par des variations des facteurs du milieu (en particulier les facteurs climatiques), de celles qui seraient le reflet d'évolution en profondeur des écosystèmes. C'est dans cette optique qu'une opération de caractérisation pédologique, édaphique et floristique (d'un coût total voisin de 3 millions de francs) est en cours de réalisation sur les 520 placettes françaises, de 1993 à 1995, dans le cadre d'un vaste projet communautaire financé à $50 \%$ par la Commission européenne. Elle devrait permettre de mieux corréler les variations de notation des houppiers et les caractéristiques du milieu, notamment celles qui déterminent l'alimentation hydrique et minérale des peuplements.

L'objectif de comparabilité des données à l'échelle européenne semble particulièrement difficile à atteindre, ainsi que l'ont montré les divers exercices internationaux d'intercalibration entre formateurs, notamment ceux organisés en France (Vosges en 1990, Innes et al., 1991 ; Sud-Est en 1991, Bouhot et al., 1992). Alors qu'il semble exister une relativement bonne cohérence entre équipes d'un même pays au fil de ces exercices, la sévérité de la notation est très différente d'un pays à l'autre. Cette hétérogénéité d'application d'un même protocole tient à une série de facteurs: difficultés de la définition d'un arbre de référence, subjectivité propre à chaque équipe de deux notateurs, densité du peuplement rendant parfois difficile l'identification complète d'un houppier, luminosité lors de la notation,... La pertinence du réseau européen réside donc essentiellement dans le suivi des variations interannuelles à différentes échelles. II est rassurant de constater de ce point de vue une bonne synchronisation des grandes variations observées par des pays riverains.

Aux niveaux national et européen, ce réseau semble donc en mesure d'atteindre effectivement l'essentiel de l'objectif qui avait motivé sa création. Les données issues du réseau de correspondants-observateurs du DSF ne permettent, la plupart du temps, qu'un suivi qualitatif des grands problèmes qui affectent les forêts françaises et risquent parfois d'alarmer un lecteur non averti. Par contre, le réseau européen a le mérite d'autoriser un bilan certes grossier dans ses indicateurs et son échelle spatiale mais reposant sur un protocole international, des grandes variations de vitalité des peuplements. II permet de relativiser, de manière relativement fiable, les appréciations subjectives et souvent pessimistes de la santé actuelle des forêts. Avec un recul supplémentaire de quelques années, ce réseau devrait permettre de repérer d'éventuelles évolutions à grande échelle de la vitalité de certaines essences, de certains types de peuplements ou de certains grands massifs, sans pouvoir néanmoins caractériser de façon précise les dysfonctionnements particuliers des écosystèmes forestiers concernés. 


\section{LE RÉSEAU RENECOFOR}

Ce troisième type de réseau vise à permettre de mieux déceler les évolutions de fond qui affectent le fonctionnement d'un nombre limité de grands types d'écosystèmes forestiers représentés en Europe, au-delà des variations conjoncturelles interannuelles, grâce au suivi détaillé et régulier d'un grand nombre de paramètres du peuplement et du milieu sur des placettes permanentes mises en place pour une trentaine d'années. Dépassant la simple observation des houppiers, il s'intéresse à la surveillance des facteurs qui paraissent le mieux caractériser le fonctionnement et les dysfonctionnements possibles de l'écosystème, tels que les ont identifiés les grands programmes de recherche des années 1980 sur les dépérissements attribués à la pollution atmosphérique à longue distance. Ce type de réseau est donc complémentaire du précédent. Ce sont d'ailleurs les mêmes groupes d'experts européens qui travaillent à l'harmonisation des protocoles utilisés.

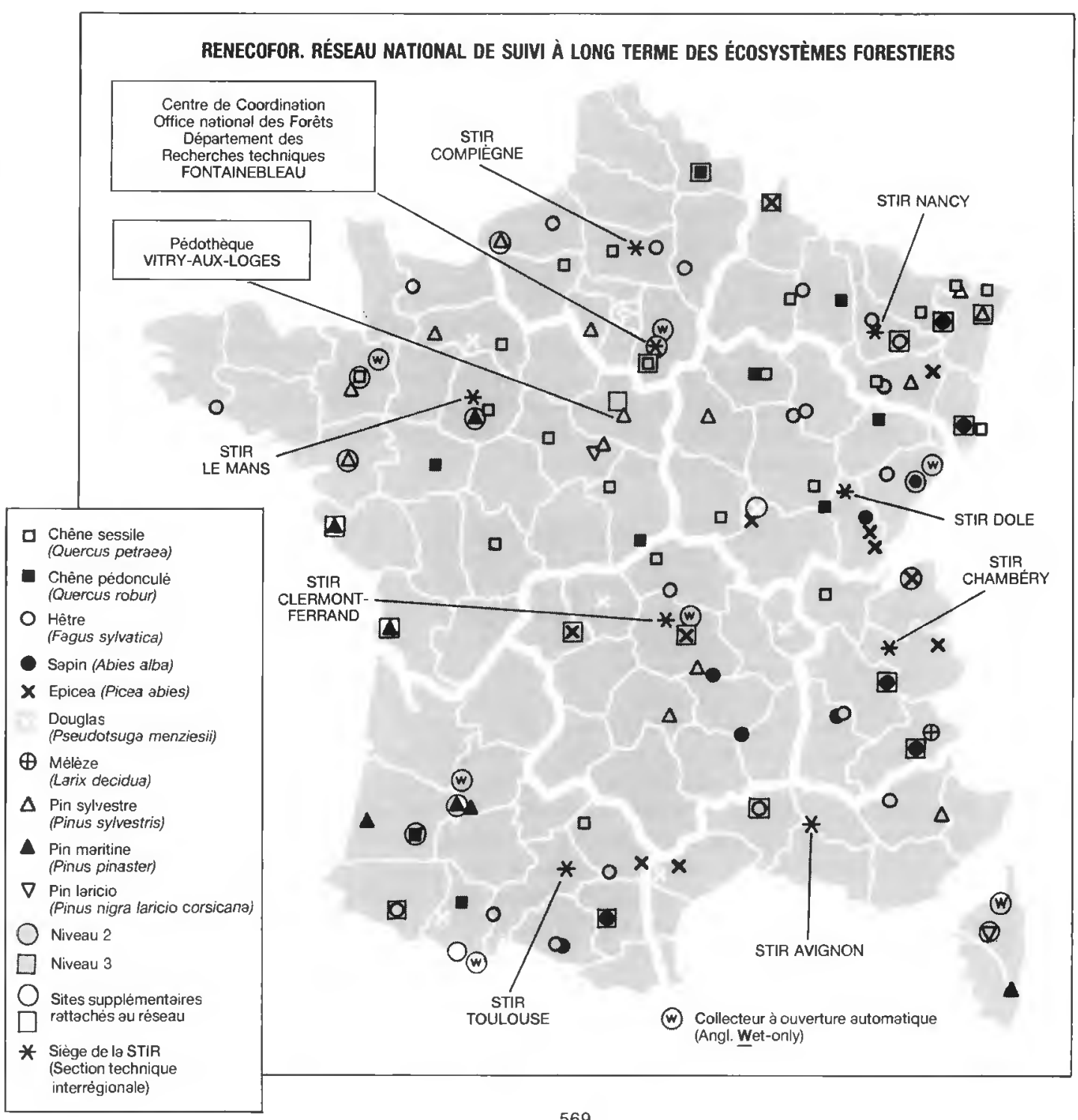




\section{Ch. BARTHOD}

La première conférence ministérielle sur la protection de la forêt en Europe (Strasbourg, décembre 1990) a officiellement fixé à ce type de réseau quatre objectifs principaux :

- obtenir des informations approfondies sur l'évolution de certains écosystèmes forestiers européens, en prenant en compte la dimension historique de l'évolution et des variations de la vitalité des forêts, des conditions stationnelles et des événements climatiques ;

- chercher à établir des corrélations entre la variation des facteurs environnementaux et la réaction des écosystèmes forestiers ;

- déterminer le niveau de charge critique en polluants susceptible de déstabiliser un type donné d'écosystème forestier ;

- permettre de mieux interpréter les résultats issus des réseaux systématiques du type du réseau européen.

Le réseau français comporte 102 placettes. Compte tenu de l'état actuel des connaissances scientifiques, la priorité a été donnée aux interactions entre le peuplement, le sol, le climat et la pollution atmosphérique, chez des peuplements d'âge moyen d'une dizaine de grandes essences sociales, menés selon des règles sylvicoles représentatives des habitudes des régions d'implantation des placettes. Implanté dans des conditions moyennes représentatives d'une région, sans traitements sylvicoles particuliers et sans prélèvements destructifs (éventuellement effectués dans la zone périphérique), il s'agit avant tout d'un réseau de surveillance (et de mesures approfondies), et non de placettes de recherche à proprement parler, même si certains projets de recherche compatibles avec le cahier des charges de ce réseau pourront probablement y être menés. À juste titre, certains experts ont fait remarquer que les évolutions se remarquent mieux dans des situations extrêmes (âges faibles ou élevés des peuplements, conditions édaphiques limitantes, ...). Néanmoins ces conditions extrêmes auraient singulièrement compliqué l'interprétation des évolutions repérées, compte tenu notamment des connaissances actuelles insuffisantes sur les variations propres à la dynamique des écosystèmes liés à des peuplements jeunes ou très âgés, ou en situation trophique difficile. Par ailleurs, il aurait été difficile d'extrapoler certaines observations au fonctionnement des écosystèmes forestiers " moyens ", gérés selon des méthodes comparables, et de chercher à en tirer des conclusions pour la gestion.

Après un état des lieux comprenant une analyse dendrochronologique, le peuplement de chaque placette sera suivi au moins annuellement par les paramètres suivants: croissance radiale, analyses foliaires, phénologie du débourrement et de la chute du feuillage, notation du houppier, présence/absence d'insectes ravageurs ou de champignons pathogènes, chute de litière. Un inventaire phytoécologique sera effectué tous les dix ans. Les prélèvements destructeurs seront effectués sur des arbres situés immédiatement à proximité de la placette de 0,5 ha. Le sol sera suivi tous les dix ans grâce à une description du profil pédologique et à des analyses chimiques d'échantillons prélevés selon un plan d'échantillonnage permettant la comparabilité des séries de données, les échantillons étant de plus archivés. Le climat sera suivi à proximité de certaines placettes par la pluviométrie, la température, l'humidité relative, et si possible le rayonnement global. L'ensemble de ces mesures constitue le niveau 1. Par ailleurs sur les placettes de niveau 2 (27 des 102 placettes), on procédera à la mesure des dépôts atmosphériques par analyse de la concentration dans les pluies hors couvert et dans les pluviolessivats (échantillonnage $h=b$ domadaire et analyses mensuelles), ainsi que dans les brouillards et gouttelettes de nuage (campagnes d'échantillonnage). Enfin, sur les placettes de niveau 3 (17 des 27 placettes précédentes), on procédera enfin à l'analyse des solutions du sol. Les entrées d'origine atmosphérique et les sorties d'éléments minéraux par lessivage sont deux termes importants du bilan minéral des écosystèmes. La mesure (ou l'estimation) des autres termes du bilan (prélèvements par les arbres et libération par la dissolution des roches) permettra à moyen terme de compléter ce bilan et de diagnostiquer l'évolution de la fertilité du sol et son incidence éventuelle sur la nutrition du peuplement. 
La décision de principe de créer un tel réseau en France, appelé réseau RENECOFOR (REseau National de suivi à long terme des ECOsystèmes FORestiers) a été prise en 1989 par Messieurs André Grammont (DERF) et Georges Touzet (ONF), sur la base d'une idée de Monsieur Maurice Bonneau (INRA), dans le cadre de la préparation de la conférence de Strasbourg par la France et la Finlande. Certains pays, comme la Suède et l'Allemagne, s'étaient engagés dans cette voie dès le début des années 1980, rejoints à la fin de la décennie par la Norvège, la Belgique et l'Autriche. En décembre 1990, les trente-et-un pays du continent européen, dont la France, se sont engagés à mettre en place de tels réseaux. En 1992, la CEE a adopté un nouveau règlement faisant de ces réseaux une priorité communautaire. Alors qu'avant 1990 la France ne disposait, contrairement à plusieurs pays européens, d'aucune structure, même régionale, de suivi approfondi des écosystèmes, elle est aujourd'hui, grâce à la mise en place du réseau RENECOFOR, le premier des grands pays forestiers de l'Union européenne à avoir constitué un réseau national unifié.

Le projet français, d'un coût total de 18,3 millions de francs pour l'installation et le bilan initial, est cofinancé par la Commission européenne, l'ONF, le ministère de l'Agriculture, le ministère de l'Environnement et l'ADEME. II a été entièrement implanté en forêt soumise au régime forestier, pour l'essentiel en forêt domaniale, compte tenu des contraintes imposées pour trente ans par le cahier des charges. II est géré par Monsieur Erwin Ulrich, ingénieur au Département des Recherches techniques de l'ONF, avec la participation active des huit sections techniques interrégionales et de 204 agents ou techniciens de l'ONF (deux personnes par placette, un titulaire et un suppléant). Après l'achèvement de la phase de mise en place des placettes, il sera sans aucun doute opportun de créer un comité de pilotage du réseau RENECOFOR, chargé d'évaluer la qualité des données récoltées, de favoriser les contacts avec les chercheurs intéressés, d'organiser l'interprétation et la valorisation des informations disponibles, et de réfléchir à l'évolution des mesures à effectuer.

\section{CONCLUSIONS}

L'ensemble de ces trois réseaux constitue une entreprise fédérative, aucun organisme forestier n'ayant les moyens d'assumer seul la responsabilité technique et scientifique de l'ensemble du dispositif. Sous la coordination technique du Département de la Santé des Forêts, les CRPF, I'ONF, les DRAF et certaines DDAF participent très activement au bon fonctionnement des deux premiers réseaux. Sous la coordination technique du Département des Recherches techniques de l'ONF, le réseau RENECOFOR fait appel aux compétences des organismes de recherches, des sections techniques interrégionales de l'ONF, de grands laboratoires d'analyses chimiques (INRA d'Arras pour les analyses de sol, INRA de Bordeaux pour les analyses foliaires, et laboratoire Wolf pour les analyses d'eau) et du Département de la Santé des Forêts. La surveillance de l'état sanitaire de la forêt traduit bien la conscience d'un intérêt commun pour une gestion patrimoniale de la qualité biologique et de la santé de la forêt.

\section{Ch. BARTHOD}

Chef du Dèpartement de la Santè des Forêts DIRECTION DE L'ESPACE RURAL ET DE LA FORÊT MINISTĖRE DE L'AGRICULTURE ET DE LA PÊCHE

1 ter, avenue de Lowendal F-75349 PARIS 07 SP 